\title{
ANÁLISE NUMÉRICA DO USO DE ANÉIS DEFLETORES INTERNOS SOBRE O ESCOAMENTO GÁS-SÓLIDO EM RISERS DE FCC
}

\author{
V. ROSSBACH ${ }^{1 *}$, R. K. DECKER ${ }^{1}$, D. NORILER ${ }^{1}$, J. UTZIG ${ }^{1,2}$, H. F. MEIER ${ }^{1}$ \\ ${ }^{1}$ Universidade Regional de Blumenau (FURB) \\ ${ }^{2}$ Universidade Federal de Uberlândia (UFU) \\ *e-mail: vivienrossbach@hotmail.com
}

\begin{abstract}
RESUMO
O escoamento gás-sólido em risers de FCC é caracterizado por uma região diluída no centro e uma região densa em catalisador, próxima às paredes. Este comportamento, caracterizado com a heterogeneidade do campo de fração volumétrica, é responsável pelo contato ineficiente entre o gás e as partículas de catalisador. Uma alternativa para homogeneizar a fração volumétrica de sólidos na região de mistura é a inserção de anéis defletores internos na região de entrada do riser. Este trabalho analisa a inserção de anéis defletores em formato de aerofólio na região de entrada do riser para redirecionar o escoamento de partículas sólidas. Para isso, foram realizadas simulações numéricas utilizando abordagem Euleriana-Euleriana, com modelo de turbulência k- $\varepsilon$. A homogeneidade da distribuição de sólidos na seção transversal do riser foi avaliada a partir do seu desvio-padrão. Com auxílio das simulações numéricas e da aplicação da ANOVA, verificou-se que o menor desvio-padrão é obtido com anéis de $10 \mathrm{~mm}$ de espessura.
\end{abstract}

\section{INTRODUÇÃO}

O craqueamento catalítico fluido (FCC - Fluid Catalytic Cracking) é um processo importante para a indústria de petróleo, convertendo gasóleo pesado de vácuo em frações leves, como a gasolina. Uma unidade de FCC consiste, basicamente, em um riser, onde ocorrem as reações químicas, e um regenerador, onde o coque depositado sobre o catalisador é removido. O gasóleo alimentado no riser é vaporizado e mistura-se com o catalisador preaquecido na região de mistura.

Devido à formação do perfil coreannulus, as partículas de catalisador acumulam-se na região das paredes, ocasionando sobrecraqueamento da alimentação próximo às paredes $\mathrm{e}$ subcraqueamento na região central do riser. Além disso, a complexidade da região de entrada causa o aumento da turbulência, levando à retromistura e à formação de curtos-circuitos no escoamento gás-sólido (GUPTA e BERRUTI, 2000; JOHNSON e DAVYDOV, 2014).

$\mathrm{O}$ uso de anéis defletores internos no riser contribui para melhorar a distribuição da fração volumétrica de sólidos nas direções axial e radial do riser e tornar o escoamento mais homogêneo. Desta forma, é possível aumentar a transferência de calor e massa no processo e melhorar a conversão e a seletividade das reações químicas de FCC (JIN, WEI e WANG, 2003). O uso de anéis defletores no interior de risers de FCC foi investigado inicialmente por Jiang, Bi e Jean (1991), com o estudo da decomposição de ozônio utilizando partículas de FCC. Exceto com velocidades mais baixas de gás, a conversão de ozônio tornou-se mais uniforme 
na direção radial e a conversão foi aumentada. Zhu, Salah e Zhou (1997) avaliaram a influência do uso de três anéis com área aberta de $70 \%, 90 \%$ e $95 \%$, respectivamente, instalados em cinco posições axiais diferentes. Os resultados mostraram maior uniformidade da distribuição de sólidos na direção radial. Para velocidades altas de gás e baixas taxas mássicas de sólidos, foram observados perfis de distribuição radial reversa, com escoamento mais diluído nas paredes que no centro. Com área aberta de $70 \%$, forma-se uma região mais densa na base que nos outros casos. A presença dos anéis também induz à formação de um perfil de velocidade axial em forma de $\mathrm{S}$.

Samruamphianskun, Piumsomboom e Chalermsinsuwan (2012) realizaram simulações numéricas bidimensionais, variando os parâmetros geométricos dos anéis e a sua posição no riser e verificaram que os fatores mais importantes são a área aberta, a espessura do anel e o número de anéis. Chalermsinsuwan, Samruamphianksun e Piumsomboon (2014) realizaram estudos variando as condições operacionais e encontraram baixa fração volumétrica e maior velocidade do gás na região dos anéis.

$\mathrm{O}$ uso de defletores na zona de mistura aproxima o escoamento do perfil empistonado, quebrando $\mathrm{o}$ annulus $\mathrm{e}$ redirecionando as partículas de catalisador para o centro do riser, o que resulta em alta conversão e diminuição do sobrecraqueamento dos produtos nas paredes. A inserção de defletores na região de alimentação traz maior uniformidade na distribuição de partículas nesta região e diminui a velocidade da região core, reduzindo assim a subconversão em produtos (JOHNSON e DAVYDOV, 2014).

Neste trabalho, avalia-se a inserção de anéis defletores abaixo da entrada de alimentação em um riser de FCC em escala de laboratório. Através de simulações numéricas e análise estatística, foram selecionadas as variáveis com maior influência sobre o escoamento e determinados os seus valores mais adequados.

\section{MODELAGEM MATEMÁTICA}

A modelagem matemática adotada segue a abordagem Euleriana-Euleriana. Tanto a fase gasosa quanto a fase sólida são consideradas contínuas. A fase sólida é tratada como um pseudofluido. O escoamento gás-sólido tridimensional e transiente foi estudado a frio. Neste trabalho, visto que o objetivo é estudar o escoamento, não foram levados em consideração os fenômenos de transferência de calor e massa, bem como as reações químicas que ocorrem no riser. A turbulência da fase gasosa foi descrita através do modelo k- $\varepsilon$ para o escoamento diluído.

A fase gasosa representa o vapor em ascensão que é alimentado na base do reator. Considerou-se que a fase gasosa é composta apenas por ar ambiente. A fase sólida é representada pelo próprio catalisador de FCC. Neste trabalho foram utilizadas partículas do Grupo A de Geldart (GELDART, 1973).

Considera-se que a fase sólida tem comportamento granular. Para calcular a temperatura granular da fase sólida, recorre-se à Teoria Cinética do Escoamento Granular (KTGF, Kinetic Theory of Granular Flow).

De acordo com Elgobashi (1994), em leitos diluídos a fração volumétrica de sólidos varia entre $10^{-7}$ e $10^{-3}$. Como a fração volumétrica de sólidos neste trabalho atinge valores máximos de cerca de $10^{-4}$, utilizou-se o modelo de arraste de Wen-Yu para descrever a interação entre as fases gasosa e sólida. Já a turbulência da fase gasosa é descrita pelo modelo $\mathrm{k}-\varepsilon$ e o movimento das partículas é dominado pela turbulência da fase gasosa. As equações adotadas encontram-se na Tabela 1 e representam o modelo matemático utilizado pelo simulador Ansys FLUENT 14.0 (Ansys FLUENT Theory Guide, 2011). 


\section{Equações da continuidade:}

Fase gás: $\frac{\partial}{\partial t}\left(f_{g} \rho_{g}\right)+\nabla \cdot\left(f_{g} \rho_{g} \mathbf{v}_{\mathbf{g}}\right)=0$

Fase sólida: $\frac{\partial}{\partial t}\left(f_{s} \rho_{s}\right)+\nabla \cdot\left(f_{s} \rho_{s} \mathbf{v}_{\mathbf{s}}\right)=0$

\section{Conservação da quantidade de movimento:}

Fase gás: $\frac{\partial}{\partial t}\left(f_{g} \rho_{g} \mathbf{v}_{\mathbf{g}}\right)+\nabla \cdot\left(f_{g} \rho_{g} \mathbf{v}_{\mathbf{g}} \mathbf{v}_{\mathbf{g}}\right)=\nabla \cdot \boldsymbol{\tau}_{\mathbf{g}}+f_{g} \rho_{g} \mathbf{g}-f_{g} \nabla p+\mathbf{F}_{\mathbf{g s}}$

Fase sólida: $\frac{\partial}{\partial t}\left(f_{s} \rho_{s} \mathbf{v}_{\mathbf{s}}\right)+\nabla \cdot\left(f_{s} \rho_{s} \mathbf{v}_{\mathbf{s}} \mathbf{v}_{\mathbf{s}}\right)=\nabla \cdot \boldsymbol{\tau}_{\mathbf{s}}+f_{s} \rho_{s} \mathbf{g}-f_{s} \nabla p-\nabla p_{s}+\mathbf{F}_{\mathbf{s g}}$

\section{Tensor tensão:}

Fase gás: $\boldsymbol{\tau}_{\mathbf{g}}=f_{g} \mu_{g}\left(\nabla \mathbf{v}_{\mathbf{g}}+\left(\nabla \mathbf{v}_{\mathbf{g}}\right)^{T}\right)-\frac{2}{3} f_{g} \mu_{g}\left(\nabla \cdot \mathbf{v}_{\mathbf{g}}\right) \mathbf{I}+f_{g} \tau_{t}$

Fase sólida: $\boldsymbol{\tau}_{\mathrm{s}}=f_{s} \mu_{s}\left(\nabla \mathbf{v}_{\mathrm{s}}+\left(\nabla \mathbf{v}_{\mathrm{s}}\right)^{T}\right)-\frac{2}{3} f_{s} \mu_{s}\left(\nabla \cdot \mathbf{v}_{\mathrm{s}}\right) \mathbf{I}$

\section{Modelo de arraste:}

Fase gás: $\mathbf{F}_{\text {draggs }}=\beta\left(\mathbf{v}_{\mathbf{s}}-\mathbf{v}_{\mathbf{g}}\right)$

Fase sólida: $\mathbf{F}_{\text {drag,sg }}=\beta\left(\mathbf{v}_{\mathbf{g}}-\mathbf{v}_{\mathbf{s}}\right)$

Modelo de Wen-Yu:

Coeficiente de transferência: $\beta=\frac{3}{4} C_{D} \frac{\left|\mathbf{v}_{\mathbf{s}}-\mathbf{v}_{\mathbf{g}}\right| f_{s} \rho_{g}}{d_{s}} f_{g}^{-2,65}$

Coeficiente de arraste:

$$
\begin{array}{ll}
\text { Para Re }>1000: & C_{D}=0,44 \\
\text { Para } \operatorname{Re} \leq 1000: & C_{D}=\frac{24}{\operatorname{Re}}\left(1+0,15 \operatorname{Re}^{0,687}\right)
\end{array}
$$

\section{Modelo KTGF:}

Viscosidade dinâmica: $\mu_{s}=\mu_{s, c o l}+\mu_{s, k i n}+\mu_{s, f r}$

Termo colisional: $\mu_{s, c o l}=\frac{4}{5} \alpha_{s} \rho_{s} d_{s} g_{0}\left(1+e_{s s}\right) \sqrt{\frac{\theta}{\pi}}$

Termo cinético: $\mu_{s, k i n}=\frac{10 \rho_{s} d_{s} \sqrt{\pi \theta}}{96 \alpha_{s}\left(1+e_{s s}\right) g_{0}}\left[1+\frac{4}{5} g_{0} \alpha_{s}\left(1+e_{s s}\right)\right]$

Termo friccional: $\mu_{s, f r}=\frac{p_{s} \sin \varphi}{2 \sqrt{I_{2 D}}}$

Pressão dos sólidos: $p_{s}=\alpha_{s} \rho_{s} \Theta+2 \rho_{s}\left(1+e_{s s}\right) \alpha_{s}^{2} g_{0} \Theta$ 
Função de distribuição radial: $g_{0}=\left[1-\left(\frac{f_{s}}{f_{s, \text { máx }}}\right)^{\frac{1}{3}}\right]^{-1}$

Viscosidade de compressão: $\lambda_{s}=\frac{4}{3} \alpha_{s} \rho_{s} d_{s} g_{0}\left(1+e_{s s}\right) \sqrt{\frac{\Theta}{\pi}}$

Temperatura granular:

$\frac{3}{2}\left[\frac{\partial}{\partial t}\left(\rho_{s} f_{s} \Theta_{s}\right)+\nabla \cdot\left(\rho_{s} f_{s} \mathbf{v}_{\mathbf{s}} \Theta_{s}\right)\right]=\left(-P_{s} \mathbf{I}+\boldsymbol{\tau}_{\mathbf{s}}\right): \nabla \mathbf{v}_{s}+\nabla \cdot\left(k_{\Theta_{s}} \nabla \Theta_{s}\right)-\gamma_{\Theta_{s}}+\phi_{g s}$

Modelo de turbulência k-\&:

Energia cinética turbulenta: $\frac{\partial}{\partial t}\left(f_{g} \rho_{g} k_{g}\right)+\nabla \cdot\left(f_{g} \rho_{g} \mathbf{v}_{\mathbf{g}} k_{g}\right)=\nabla \cdot\left(f_{g} \frac{\mu_{t, g}}{\sigma_{k}} \nabla k_{g}\right)+f_{g} G_{k, g}-f_{g} \rho_{g} \varepsilon_{g}$

Dissipação de energia cinética turbulenta:

$\frac{\partial}{\partial t}\left(f_{g} \rho_{g} \varepsilon_{g}\right)+\nabla \cdot\left(f_{g} \rho_{g} \mathbf{v}_{\mathbf{g}} \varepsilon_{g}\right)=\nabla \cdot\left(f_{g} \frac{\mu_{t, g}}{\sigma_{\varepsilon}} \nabla \varepsilon_{g}\right)+f_{g} \frac{\varepsilon_{g}}{k_{g}}\left(C_{1 \varepsilon} G_{k, g}-C_{2 \varepsilon} \rho_{g} \varepsilon_{g}\right)$

Produção de energia cinética turbulenta:

Dissipação da flutuação de energia:

Viscosidade do gás: $\mu_{g}=\mu_{l, g}+\mu_{t, g}$

Viscosidade turbulenta: $\mu_{t, g}=\rho_{g} C_{\mu} \frac{k_{g}^{2}}{\varepsilon_{g}}$

\section{MATERIAIS E MÉTODOS}

Para a realização das simulações numéricas foram testadas três malhas, contendo, respectivamente, 265000, 380000 e 578000 volumes de controle. A malha de 380000 volumes de controle foi escolhida através da verificação da estabilidade da solução numérica.

As medidas geométricas do riser e dos anéis são indicadas na Figura 1. A partir do planejamento fatorial $2^{4-1}$, foram realizadas simulações numéricas com diversas configurações de anéis defletores. Estas configurações são apresentadas na Figura 2.

Por analogia com o formato aerodinâmico de um aerofólio, foi proposto o formato de anel defletor apresentado na
Figura 1. De acordo com Anderson Jr. (2015), o formato do aerofólio retarda o descolamento da camada limite laminar e a formação de vórtices, responsáveis pela retromistura e por curtos-circuitos, além de amenizar as diferenças de pressão ao longo da sua área. Quanto mais suave é a curva do aerofólio, menor é a queda de pressão.

O planejamento experimental $2^{4-1}$, apresentado na Tabela 3, foi proposto para avaliar a influência sobre o escoamento de quatro variáveis: a espessura do anel $\left(\mathrm{X}_{\mathrm{A}}\right)$, o espaçamento entre anéis $\left(X_{B}\right)$, o número de anéis $\left(\mathrm{X}_{\mathrm{C}}\right)$ e a presença de um anel abaixo da entrada de catalisador $\left(\mathrm{X}_{\mathrm{D}}\right)$.

A partir do planejamento experimental, foram realizadas simulações numéricas utilizando o modelo matemático 
proposto na Tabela 1 com o código de CFD FLUENT 14.0 da Ansys. Como as amostras são de tamanhos iguais, calcula-se o desviopadrão $(\sigma)$ das mesmas para avaliar a homogeneidade da distribuição da fração volumétrica de sólidos na seção transversal, como variável de resposta do planejamento. As amostras utilizadas são obtidas a partir de dados das simulações numéricas em três alturas - 1,05m, 1,2m e 1,35m - conforme indicado na Figura 1.

Figura 1 - Medidas geométricas do riser.

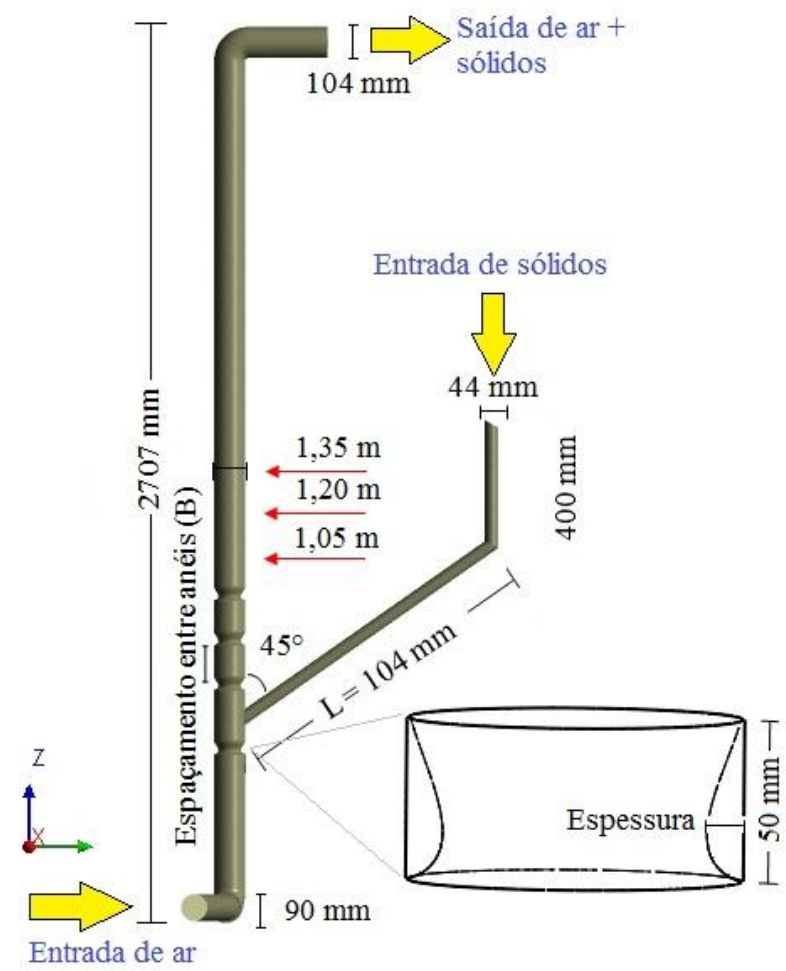

\section{RESULTADOS E DISCUSSÃO}

As geometrias construídas a partir do planejamento experimental são apresentadas na Figura 2. Foram resolvidos 15 segundos de simulação numérica para cada caso, com passo de tempo de $1 \times 10^{-3} \mathrm{~s}$. As propriedades físicas e operacionais do sistema são descritas na Tabela 2. Nas paredes, adotou-se condição de não-deslizamento para o gás e livre deslizamento para as partículas.
Com os dados obtidos das simulações numéricas, realizou-se a análise estatística dos dados pela ANOVA (Analisys of Variance).

Tabela 2 - Propriedades físicas e condições operacionais do escoamento.

Massa específica do

catalisador

$1500 \mathrm{~kg} / \mathrm{m}^{3}$

Diâmetro das partículas de

catalisador

$67 \mu \mathrm{m}$

Velocidade do gás

$5,6 \mathrm{~m} / \mathrm{s}$

Vazão mássica de sólidos

Fluxo mássico de sólidos

Razão de carga

$0,0086 \mathrm{~kg} / \mathrm{s}$

$1,41 \mathrm{~kg} / \mathrm{m}^{2} \mathrm{~s}$

Carregamento mássico

$0,181 \mathrm{~kg} \mathrm{só} 1 / \mathrm{m}^{3}$ gás

$0,151 \mathrm{~kg}$ sól $/ \mathrm{kg}$ gás

A Tabela 3 mostra o planejamento fatorial $2^{4-1}$ com o desvio-padrão médio das três amostras. O menor desvio-padrão foi obtido no Caso 3, indicando maior homogeneidade da distribuição de partículas sólidas na seção transversal.

Tabela 3 - Planejamento experimental $2^{4-1}$.

\begin{tabular}{cccccc}
\hline Caso & \multicolumn{4}{c}{ Variáveis controladas } & $\begin{array}{c}\text { Variável } \\
\text { de } \\
\text { resposta }\end{array}$ \\
& $\mathrm{X}_{\mathrm{A}}$ & $\mathrm{X}_{\mathrm{B}}$ & $\mathrm{X}_{\mathrm{C}}$ & $\mathrm{X}_{\mathrm{D}}$ & $\sigma \times 10^{-3}$ \\
Sem & - & - & - & - & 5,047 \\
1 & 10 & 60 & 2 & 0 & 4,737 \\
2 & 15 & 60 & 2 & 1 & 4,730 \\
3 & 10 & 100 & 2 & 1 & 4,711 \\
4 & 15 & 100 & 2 & 0 & 4,803 \\
5 & 10 & 60 & 3 & 1 & 4,790 \\
6 & 15 & 60 & 3 & 0 & 4,732 \\
7 & 10 & 100 & 3 & 0 & 4,757 \\
8 & 15 & 100 & 3 & 1 & 4,804 \\
\hline
\end{tabular}

Através do software Statistica, aplicou-se a ANOVA fatorial sobre os dados do planejamento experimental, para estimar a influência das variáveis controladas sobre a variável de resposta. A partir desta análise obteve-se a Equação 26, que prediz os efeitos das variáveis e suas interações sobre o desviopadrão da fração volumétrica de sólidos na seção transversal do riser. 
$\sigma \times 10^{-3}=4,758-0,01275 \mathrm{X}_{\mathrm{A}}(10 \mathrm{~mm})$

$-0,00925 \mathrm{X}_{\mathrm{B}}(60 \mathrm{~mm})-0,01075 \mathrm{X}_{\mathrm{C}}(2)$

$-0,75 \times 10^{-3} \mathrm{X}_{\mathrm{D}}(0)-0,012 \mathrm{X}_{\mathrm{A}} \mathrm{X}_{\mathrm{B}}$

$-0,001 \mathrm{X}_{\mathrm{B}} \mathrm{X}_{\mathrm{C}}+0,0255 \mathrm{X}_{\mathrm{C}} \mathrm{X}_{\mathrm{D}}$

A Equação 26 mostra que as variáveis $\mathrm{X}_{\mathrm{A}}$ e $\mathrm{X}_{\mathrm{C}}$ e as interações entre os efeitos $\mathrm{X}_{\mathrm{A}} \mathrm{X}_{\mathrm{B}}$ e $X_{C} X_{D}$ exercem maior influência sobre $o$ desvio-padrão da fração volumétrica de sólidos na seção transversal.

Figura 2 - Geometrias do riser.

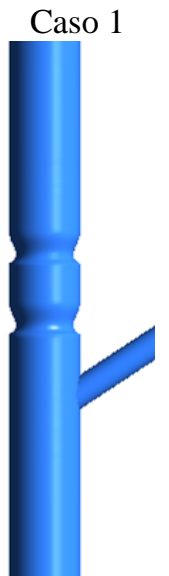

Caso 5
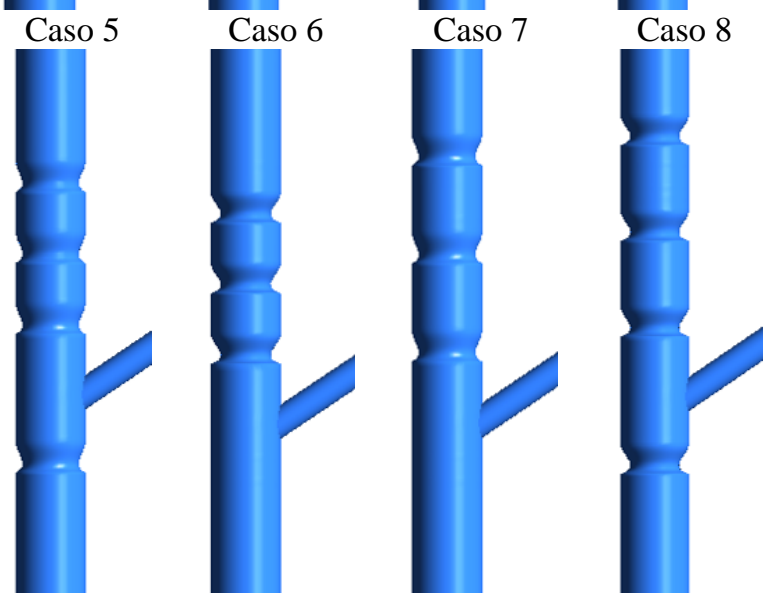

A Figura 3 mostra a superfície de resposta das variáveis $X_{A}$ e $X_{D}$ sobre $o$ desvio-padrão, onde os menores valores de desvio-padrão são obtidos com a utilização de dois anéis com $10 \mathrm{~mm}$ de espessura. As interações entre efeitos $X_{A} X_{B}$ e $X_{C} X_{D}$ são apresentadas nas Figuras 4-(a) e 4-(b).
Figura 3 - Superfície de resposta das variáveis $\mathrm{X}_{\mathrm{A}}$ e $\mathrm{X}_{\mathrm{C}}$ sobre o desvio-padrão.

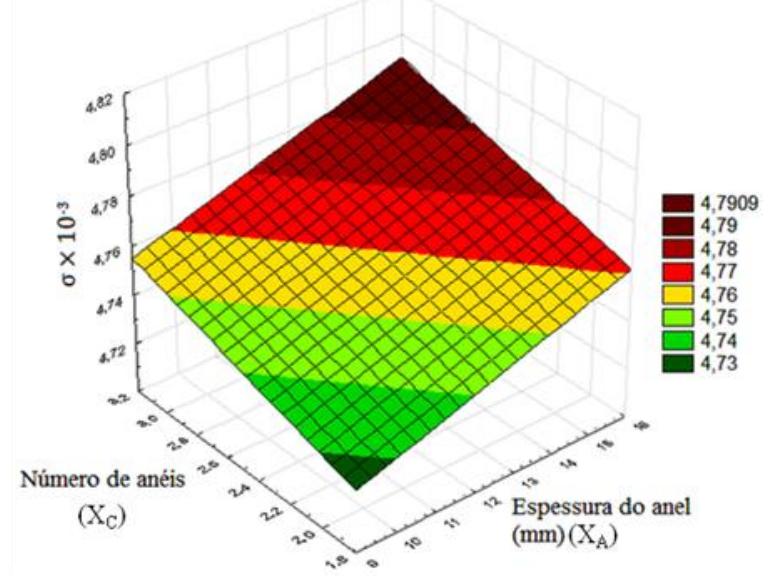

Figura 4 - Superfícies de resposta das interações $\mathrm{X}_{\mathrm{A}} \mathrm{X}_{\mathrm{B}}$ e $\mathrm{X}_{\mathrm{C}} \mathrm{X}_{\mathrm{D}}$ sobre o desvio-padrão.

(a)

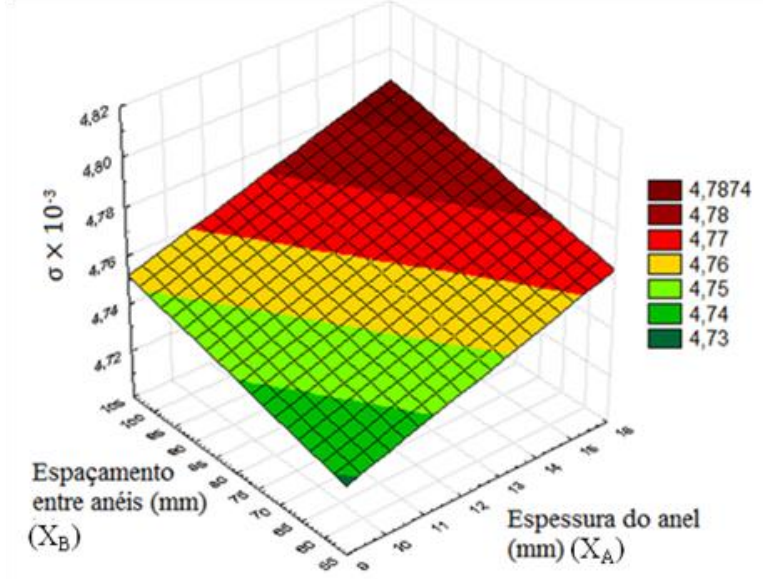

(b)

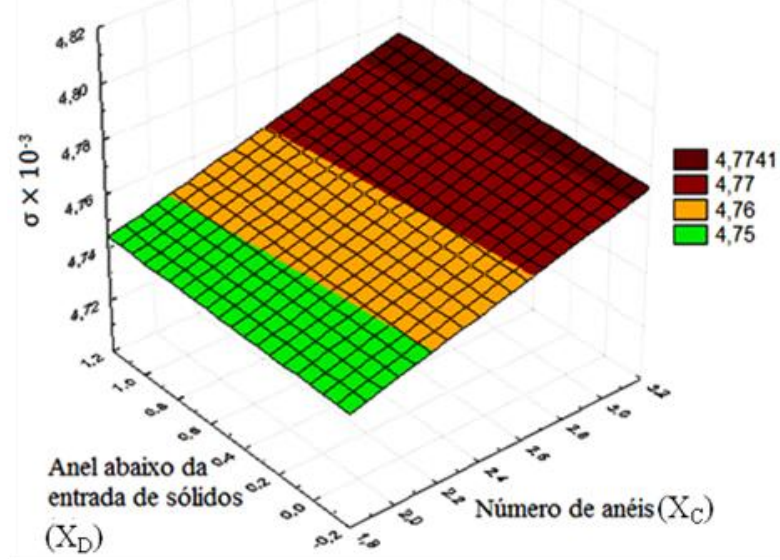


Os menores valores de desvio-padrão são obtidos com anéis de $10 \mathrm{~mm}$ com espaço de cerca de $65 \mathrm{~mm}$ entre si e os valores mínimos das variáveis $\mathrm{X}_{\mathrm{C}}$ e $\mathrm{X}_{\mathrm{D}}$ produzem os melhores resultados. Desta forma, a inserção de dois anéis produz um bom resultado, enquanto a presença do anel abaixo da entrada de sólidos tem efeito negativo sobre o desviopadrão. Na Figura 5, observa-se a distribuição axial da fração volumétrica de sólidos para o Caso 3, que apresentou o melhor resultado quanto à distribuição radial de sólidos e ao seu desvio-padrão. $\mathrm{O}$ anel localizado abaixo da entrada de sólidos tem a função de redirecionar o escoamento do gás, o que auxilia na distribuição das partículas ao longo da seção transversal do riser.

Na Figura 5, observa-se a tendência de escoamento de sólidos na parede oposta à sua entrada, porém as partículas são bem distribuídas ao longo do riser. A utilização de anéis de $10 \mathrm{~mm}$ melhorou a distribuição de sólidos, enquanto a maior espessura resultou em pior distribuição. Os resultados permitem ainda constatar que o regime de escoamento é de fato diluído.

A tendência de os sólidos escoarem na parede oposta à sua entrada pode ser atribuída à curva de entrada de gás ou à própria entrada de sólidos. Para avaliar este efeito, é necessário realizar testes sem a curva na entrada de gás. Estes testes, bem como a construção dos anéis defletores para obtenção de dados experimentais, estão em andamento e serão publicados em trabalhos futuros. A validação do modelo matemático com dados experimentais permitirá, ainda, a ampliação de escala do experimento, visando à escala industrial.

A simples análise do desvio-padrão não garante a homogeneidade da distribuição de sólidos no riser, tornando-se necessária a análise visual dos resultados. Desta forma, os dados estatísticos devem ser confrontados com dados visuais de simulações numéricas para se identificar a melhor distribuição de fração volumétrica dentre os casos simulados.

Figura 5 - Contornos de fração volumétrica de sólidos na direção axial para o Caso 3 .

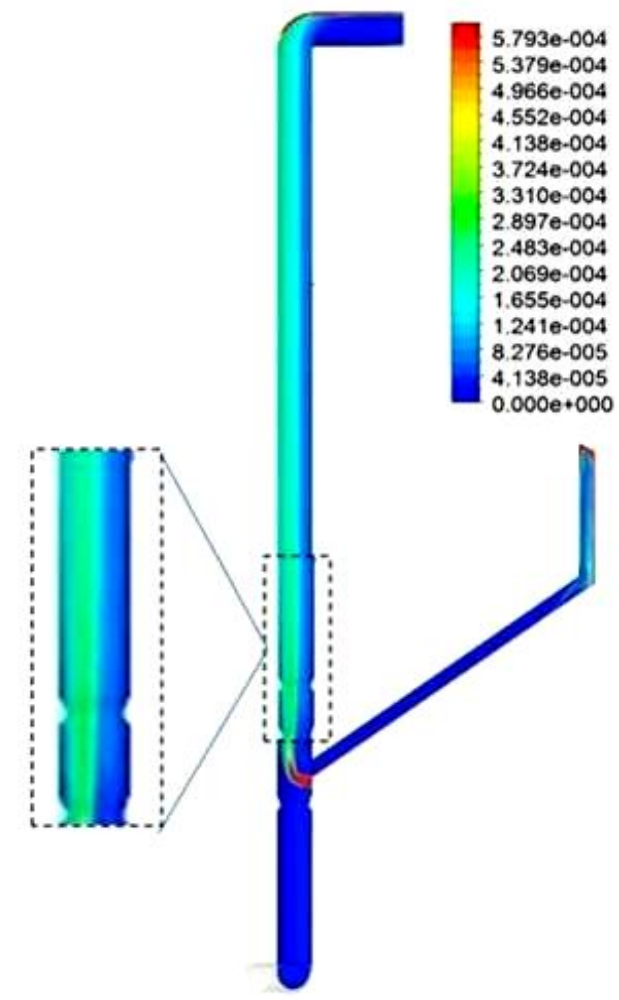

A análise dos contornos de fração volumétrica na seção transversal do riser, apresentada na Figura 6, auxilia na identificação do experimento com melhor distribuição de sólidos. Os Casos 3 e 5 têm distribuições de sólidos bastante semelhantes, o que não é indicado pela análise de desvio padrão. Em relação ao Caso 2, no entanto, a comparação entre o desvio-padrão calculado e a análise visual dos contornos fornecem as mesmas conclusões.

O padrão de distribuição mantém-se ao longo da direção axial na região medida. Logo, os anéis aumentam a homogeneidade da distribuição de sólidos nas direções axial e radial. Ao se afastarem dos anéis, as partículas sólidas tendem a se distribuir do centro em direção às paredes. Nos casos 2, 3 e 5 , este comportamento é mais bem observado. 
Figura 6 - Contornos de fração volumétrica de sólidos na seção transversal.

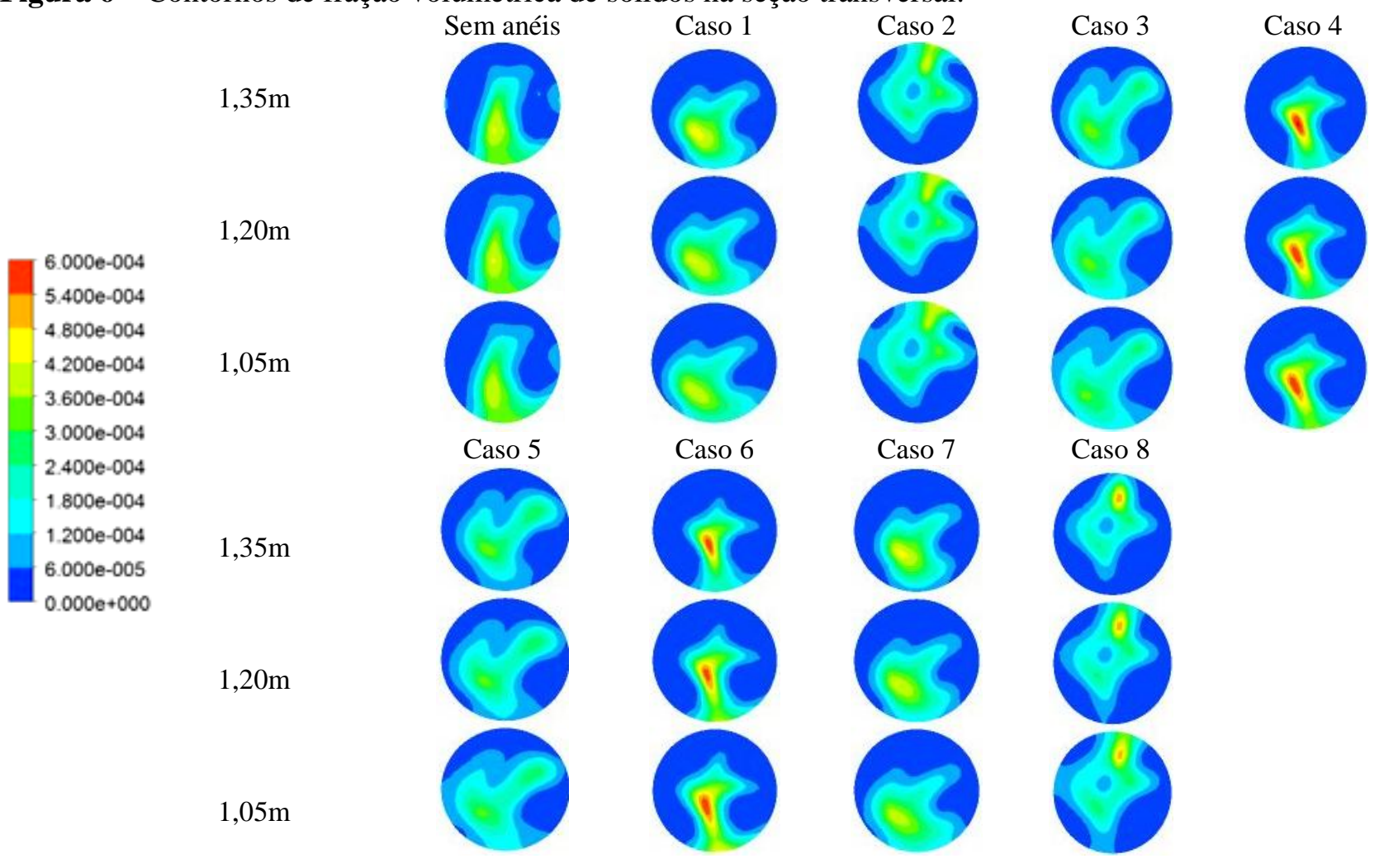

A Tabela 4 mostra a perda de pressão em cada caso, em comparação com o riser sem anéis.

Tabela 4 - Perda de pressão no riser

\begin{tabular}{lr}
\hline Caso & $\boldsymbol{A p}(\mathbf{P a})$ \\
Sem anéis & 83,96 \\
1 & 90,18 \\
2 & 179,00 \\
3 & 101,32 \\
4 & 141,17 \\
5 & 112,09 \\
6 & 168,44 \\
7 & 96,78 \\
8 & 215,84 \\
\hline
\end{tabular}

A perda de pressão no riser é proporcional ao número de anéis e à espessura do anel. Nos Casos 1, 3, 5 e 7, onde a espessura do anel é de $10 \mathrm{~mm}$, a perda de pressão é baixa em comparação com o riser sem anéis. Já nos demais casos, a perda de pressão é maior. O Caso 3, que apresentou a melhor distribuição de sólidos, tem uma perda de pressão consideravelmente baixa. Estes dados condizem com a teoria apresentada em Anderson Jr. (2015) para o escoamento na superfície do aerofólio. No riser com anéis defletores estudado, da mesma forma como em um aerofólio, a menor espessura do anel causa menor queda de pressão.

\section{CONCLUSÕES}

Através da análise dos dados obtidos, conclui-se que os anéis defletores em formato de aerofólio são capazes de redistribuir o escoamento gás-sólido e homogeneizar a distribuição de partículas no riser.

A aplicação da ANOVA mostrou que a espessura do anel $\left(\mathrm{X}_{\mathrm{A}}\right)$, o número de anéis $\left(\mathrm{X}_{\mathrm{C}}\right)$ e as interações $\mathrm{X}_{\mathrm{A}} \mathrm{X}_{\mathrm{B}}$ e $\mathrm{X}_{\mathrm{C}} \mathrm{X}_{\mathrm{D}}$ são as variáveis com maior influência sobre a distribuição de sólidos no riser. A comparação entre os resultados estatísticos e os contornos de fração volumétrica de sólidos 
obtidos a partir das simulações permite identificar o Caso 3 como a melhor configuração geométrica para o riser com anéis. A configuração geométrica do Caso 3 apresentou boa distribuição de sólidos nas direções radial e axial e uma baixa perda de pressão ao longo do riser.

A assimetria do escoamento deve ser verificada através da realização de simulações numéricas sem a curva de entrada de gás. Desta forma, será possível confirmar se este efeito é causado pela curva de entrada de gás ou pela própria entrada de sólido.

\section{NOMENCLATURA}

\section{Letras latinas:}

$\mathrm{C}_{1 \varepsilon} \quad$ constante do modelo k- $\varepsilon$ igual a 1,44 [-]

$\mathrm{C}_{2 \varepsilon} \quad$ constante do modelo $\mathrm{k}-\varepsilon$ igual a 1,92 $[-]$

$\mathrm{C}_{3 \varepsilon} \quad$ constante do modelo k- $\varepsilon$ igual a 1,2 [-]

$C_{D} \quad$ coeficiente de arraste [-]

$\mathrm{C}_{\mu} \quad$ constante do modelo $\mathrm{k}-\varepsilon$ igual a 0,09 [-]

d diâmetro [m]

$e_{s s} \quad$ coeficiente de restituição igual a 0,9 $[-]$

$f \quad$ fração volumétrica [-]

F força de interação [N]

g aceleração da gravidade igual a $9,87 \mathrm{~m} / \mathrm{s}^{2}\left[\mathrm{~m}^{2} \mathrm{~s}^{-1}\right]$

$g_{0} \quad$ função de distribuição radial [-]

$\mathrm{G}_{k, g} \quad$ termo de geração turbulenta $\left[\mathrm{kg} \mathrm{m}^{-1} \mathrm{~s}^{-3}\right]$

I tensor unitário [Pa]

I módulo do tensor unitário $[\mathrm{Pa}]$

$k \quad$ energia cinética turbulenta $\left[\mathrm{m}^{2} \mathrm{~s}^{-2}\right]$

$\mathrm{k}_{\Theta s} \quad$ difusão de energia da fase sólida $\left[\mathrm{m}^{2} \mathrm{~s}^{-1}\right]$

$p_{s} \quad$ pressão dos sólidos $[\mathrm{Pa}]$

$p$ pressão [Pa]

$\mathrm{P}^{k}$ produção de energia cinética turbulenta $\left[\mathrm{Pa} \mathrm{s}^{-1}\right]$
Re número de Reynolds [-]

$U \quad$ velocidade ponderada $\left[\mathrm{m} \mathrm{s}^{-1}\right]$

$\mathbf{v} \quad$ velocidade $\left[\mathrm{m} \mathrm{s}^{-1}\right]$

$\mathrm{X}_{\mathrm{i}}$ variáveis do planejamento experimental [-]

\section{Letras gregas:}

$\begin{array}{ll}\alpha & \text { ângulo interno de fricção }\left[{ }^{\circ}\right] \\ \beta & \text { coeficiente de transferência entre as } \\ & \text { fases }\left[\mathrm{kg} \mathrm{m}^{-3} \mathrm{~s}^{-1}\right] \\ \gamma & \text { dissipação da flutuação de energia }\end{array}$

dissipação da flutuação de energia $\left[\mathrm{kg} \mathrm{m}^{-1} \mathrm{~s}^{-3}\right]$

$\varepsilon$ dissipação de energia cinética turbulenta $\left[\mathrm{m}^{2} \mathrm{~s}^{-3}\right]$

$\Theta \quad$ temperatura granular $\left[\mathrm{m}^{2} \mathrm{~s}^{-2}\right]$

$\theta$ ângulo entre velocidade média da partícula e velocidade média relativa $\left[{ }^{\circ}\right]$

$\mu \quad$ viscosidade $\left[\mathrm{kg} \mathrm{m}^{-1} \mathrm{~s}^{-1}\right]$

$\lambda \quad$ viscosidade mássica $\left[\mathrm{kg} \mathrm{m}^{-1} \mathrm{~s}^{-1}\right]$

$\pi \quad$ constante pi [-]

$\rho \quad$ massa específica $\left[\mathrm{kg} \mathrm{m}^{-3}\right]$

$\sigma \quad$ desvio-padrão [-]

$\tau$ tensor tensão turbulento [Pa]

$\tau_{t} \quad$ tensor tensão turbulenta [Pa]

$\varphi \quad$ angulo de contato $\left[{ }^{\circ}\right]$

$\phi_{g s} \quad$ termo de interação entre as fases $\left[\mathrm{kg} \mathrm{m}^{-1} \mathrm{~s}^{-3}\right]$

\section{Subscritos:}

$\begin{array}{ll}g & \text { fase gás } \\ s & \text { fase sólida } \\ \text { drag } & \text { arraste } \\ \text { col } & \text { colisional } \\ \text { kin } & \text { cinético } \\ f r & \text { friccional } \\ \text { máx } & \text { máximo } \\ l & \text { laminar } \\ t & \text { turbulento } \\ \text { drift } & \text { tração }\end{array}$




\section{REFERÊNCIAS}

ANDERSON JR., J. E. Fundamentos de

Engenharia Aeronáutica: introdução ao vôo.

7. ed. São Paulo: McGraw-Hill LLC, 2015. $925 \mathrm{p}$.

ANSYS FLUENT Theory Guide. Canonsburg (PA): Ansys, Inc., 2011.

CHALERMSINSUWAN, B.;

SAMRUAMPHIANSKUN,T.;

PIUMSOMBOON, P. Effect of operating parameters inside circulating fluidized bed reactor riser with ring baffles using CFD simulation and experimental design analysis.

Chemical Engineering Research and Design, v. 92, p. 2479-2492, 2014.

ELGOBASHI, S. E. On predicting particleladen turbulent flows. Journal of Applied Scientific Research, v. 52, n. 4, p. 309-329, 1994.

GELDART, D. Types of gas fluidization. Powder Technology, v. 7, p. 285-292, 1973.

GUPTA, S. K.; BERRUTI, F. Evaluation of the gas-solid suspension density in CFB risers with exit effects. Powder Technology, v. 18, p. 21-31, 2000.

JIANG, P.; BI, H.; JEAN, R. Baffle effects on performance of catalytic circulating fluidized bed reactor. AIChE Journal, v. 37, p. 13921400, 1991.

JIN, Y.; WEI, F.; WANG, Y. Effect of internal tubes and baffles. In: YANG, W. C. Handbook of fluidization and fluid-particle systems. New York: Marcel Dekker, 2003. Cap. 7, p. 851.

JOHNSON, D. R.; DAVYDOV, L. Baffles for improving riser hydrodynamics. US8877132B2, 4 nov. 2014.
SAMRUAMPHIANSKUN, $\quad$ T; ; PIUMSOMBOON, $P$; CHALERMSINSUWAN, B. Effect of ring baffle configurations in a circulating fluidized bed riser using CFD simulation and experimental design analysis. Chemical Engineering Journal, v. 210, p. 237-251, 2012.

ZHU, J. X.; SALAH, M.; ZHOU, Y. M. Radial and axial voidage distributions in circulating fluidized bed with ring-type internals. Japanese Journal of Chemical Engineering, v. 30, n. 5, p. 928-937, 1997.

\section{AGRADECIMENTOS}

Os autores agradecem o apoio financeiro da PETROBRAS para a realização desta pesquisa, através do termo de cooperação 0050.0070 334.11.9. 